\title{
Intrinsic Homology-Sensing and Assembling Property of Chromatin Fiber
}

\author{
Jun-ichi Nishikawa, Yasutoshi Shimooka and \\ Takashi Ohyama \\ Additional information is available at the end of the chapter \\ http://dx.doi.org/10.5772/56548
}

\section{Introduction}

The first meiotic prophase is divided into five sequential stages, referred to as leptotene, zygotene, pachytene, diplotene and diakinesis. Chromosome pairing is initiated during the late leptotene and early zygotene. As the stage progresses, the four chromatids are arranged and form two distinct pairs of sister chromatids, and the two chromatids in each pair are tightly aligned along their entire lengths. The synaptonemal complex (SC) is fully formed at the pachytene stage. Although such morphological changes in chromosomes and the mechanism of recombination have been well studied, we do not still understand how each chromosome recognizes and approaches its matching mate or homologue and the mechanism by which it interacts and pairs with its homologue. Regarding the latter phenomenon, double-stranded (ds) DNA has long been considered to have the ability to distinguish self and non-self. Indeed, this property of DNA was predicted about 60 years ago (Jehle, 1950; Yos et al., 1957) and was directly demonstrated a half-decade ago, when dsDNA molecules were proved to have sequence-sensing and self-assembly properties (Inoue et al., 2007). In the following two years, other studies describing the presence of such properties in DNA were reported (Baldwin et al., 2008; Danilowicz et al., 2009). A more recent study has revealed that nucleosomes retain this DNA sensing property and can self-assemble (Nishikawa and Ohyama, 2013). In this chapter, we define the self-association of the same dsDNA molecules as DNA self-assembly and that of nucleosomes with identical DNA as nucleosome self-assembly. And, we will discuss the recent findings on DNA self-assembly and nucleosome self-assembly and their relationships, as putatively significant mechanisms in the early stage in meiosis. 


\section{Self-assembly in biological systems}

Self-assembly is the autonomous organization of constituents into higher-order structures or assemblages, in which disordered pre-existing components form organized architectures as a consequence of specific, local interactions among the components, without external intervention. This phenomenon is widely found in the natural world, and is a fundamental mechanism in biological systems (Whitesides and Grzybowski, 2002). A characteristic of biological selfassembly is the variety and complexity of the functions of the resulting structures (Kushner, 1969; Perham, 1975). We see such examples in lipid bilayer formation, base pairing, protein folding, protein-protein interactions, including the formation of quaternary structures, protein-nucleic acid interactions, flagella formation from flagellin, actin assembly, microtubule and microfibril formation, and virus, organ and cell assembly (Kushner, 1969; Whitesides and Grzybowski, 2002; Mueller et al., 1962; Watson and Crick, 1953; Oosawa and Asakura, 1975; Asakura, 1968; Horváth et al., 1949; Miki-Noumura and Mori, 1972; Fraser et al., 1976; FraenkelConrat and Williams, 1955). Generally, self-assembly occurs by the tendency of a system to move toward the state of minimum free energy, and the assemblages are the result of the thermodynamic equilibrium, which is determined by various conditions, including temperature, $\mathrm{pH}$, pressure, the concentrations and/or chemical potentials of various molecules and ions (Oosawa and Asakura, 1975) and London-van der Waals forces (Jehle, 1963).

\section{DNA can sense homology and self-assemble}

The possibility of a nucleotide sequence-dependent, selective interaction between dsDNA fragments was first studied theoretically (Kornyshev and Leikin, 2001). The following scenario was depicted: sequence-dependent twist modulation leads to axial variation of the local helical pitch, which allows an electrostatically favorable alignment of two DNA fragments; as the result, only DNA with homologous sequences can have negatively charged strands facing positively charged grooves over a large juxtaposition length, and nonhomologous sequences cannot align well because they require higher energy for juxtaposition. In another model, proposed over 40 years ago, the homology recognition is based on non-Watson-Crick hydrogen bond interactions occurring between bases in the major or minor grooves (McGavin, 1971). In still another model, proposed over 50 years ago, homology recognition is based on correlations in the fluctuating polarizations of nearby identical molecules (the London-van der Waals force) (Yos et al., 1957).

The first experimental evidence proving that DNA can sense homology and self-assemble was obtained in 2007 (Inoue et al., 2007). Electrophoretic analyses of thirteen different dsDNA molecules with 100 - 800 bp lengths and an atomic force microscopy (AFM) analysis revealed that in a solution composed of heterogeneous DNA species, DNA molecules preferentially interact with the molecules bearing an identical sequence and length, and they can form assemblages (Fig. 1A). This phenomenon efficiently occurs in the presence of physiological concentrations of $\mathrm{Mg}^{2+}$ ions, usually several mM. Nanomolar DNA concentrations also seem 
to be a prerequisite for the stable formation of self-assemblages. Interestingly, curved DNA and DNA with an A-form-like conformation also exhibited the self-assembling property. Thus, this phenomenon is not specific to the usual B-form DNA, but seems to be general for all kinds of dsDNA.

\section{A}

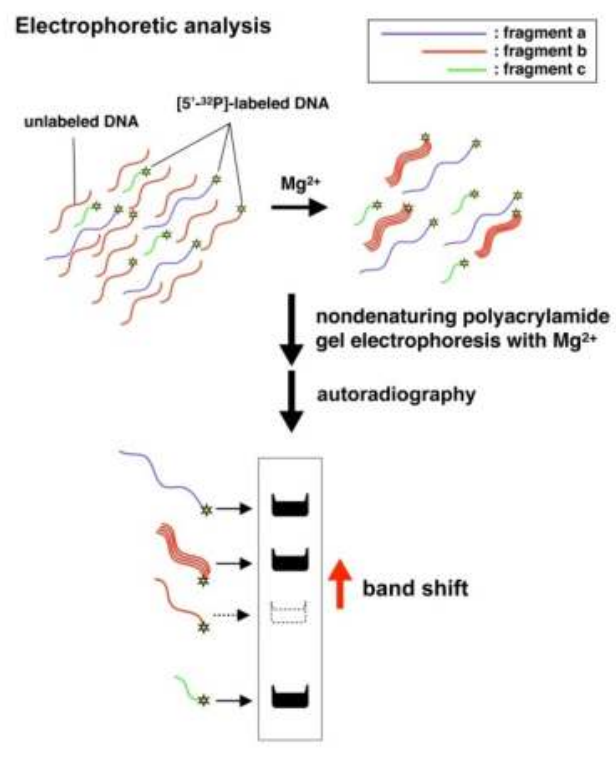

B

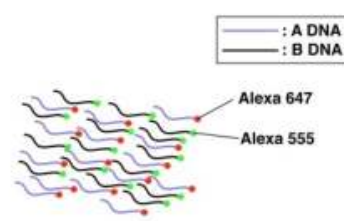

mixture of two different DNA molecules

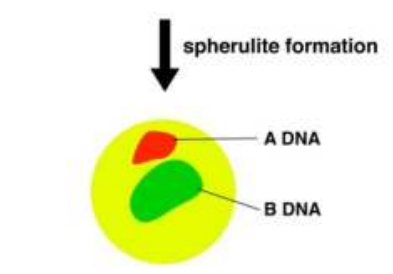

segregation of DNA molecules

\section{AFM analysis}

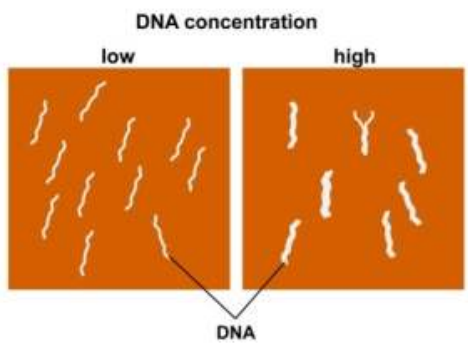

\section{C}
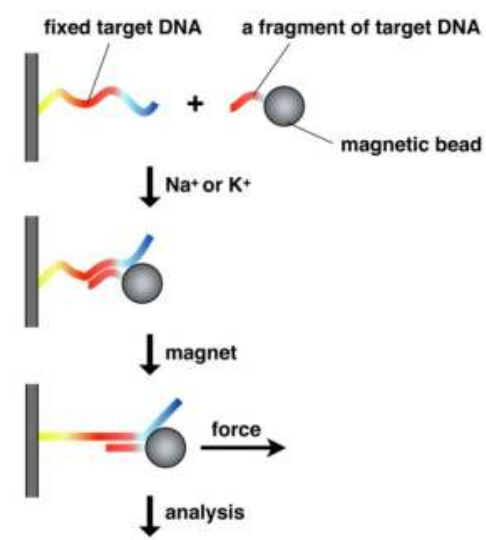

position, stability, force etc.

Figure 1. Three different experimental approaches proved intrinsic homology-sensing and assembling property of dsDNA. (A) Electrophoretic and AFM analyses (Inoue et al., 2007). (B) Spherulite analysis (Baldwin et al., 2008). (C) Parallel single molecule magnetic tweezers-based assay (Danilowicz et al., 2009). 
In 2008 (Baldwin et al., 2008), another experimental approach confirmed this homology-sensing and pairing ability of dsDNA. In this approach, $5^{\prime}$-amine-modified-DNAs with $\sim 300$ bp lengths were labeled with either Alexa Fluor 555, a green fluorescent tag, or Alexa Fluor 647, a far-red fluorescent tag, condensed into aggregates, mounted on microscope slides, equilibrated for two weeks, and subjected to a confocal microscopy analysis. Interestingly, segregation of the two kinds of DNA was observed within each spherulite (discrete liquid-crystalline aggregate) (Fig. 1B). Although the experimental conditions seemed to be far from in vivo conditions (extremely high concentrations of $\mathrm{NaCl}(>0.5 \mathrm{M})$ and DNA were used to generate spherulites), this study succeeded in extracting a hidden property of dsDNA.

In 2009, using phage $\lambda$ DNA and a parallel single molecule magnetic tweezers-based assay, an experiment further confirmed the presence of homologous dsDNA pairing (Danilowicz et al., 2009) (Fig. 1C). This study showed that pairing can occur even in the absence of divalent cations or crowding agents. Specifically, the pairing occurred in the presence of more than 50 $\mathrm{mM} \mathrm{Na}^{+}$or $\mathrm{K}^{+}$(the effect of the latter was generally smaller than that of the former). In $10 \mathrm{mM}$ $\mathrm{Na}^{+}$, homologous pairing between $\lambda$ DNA and its $5 \mathrm{~kb}$ fragment was not detected. However, significant homologous pairing was detected between the two at $10 \mathrm{mM} \mathrm{Mg}^{2+}$. Although a 10 $\mathrm{mM} \mathrm{Mg}{ }^{2+}$ concentration may be slightly higher than the in vivo concentration (Terasaki and Rubin, 1985), the essence of this finding was the same as that in the report by Inoue et al. (2007). Furthermore, the detected pairs were stable against thermal forces (up to $\sim 50^{\circ} \mathrm{C}$ ) and shear forces up to $10 \mathrm{pN}$. Homologous pairing was detected at 1-3 nM DNA, which was also consistent with the report by Inoue et al. (2007).

In conclusion, the three above-mentioned studies proved that dsDNA has homology-sensing ability and can self-assemble, but the mechanism underlying the phenomenon remains enigmatic. The next issue to be discussed is whether nucleosomes, the fundamental units of chromatin (Luger et al., 1997), have sequence-dependent self and non-self discrimination properties. Regarding this, to our knowledge, no report has ever been presented. Before moving on to this subject, let's review the structures of chromatin and nucleosomes.

\section{Nucleosomes, the fundamental units of chromatin}

Chromatin provides the structural basis for all nuclear events involving DNA, such as replication, transcription, repair and recombination (Ransom et al., 2010; Svejstrup, 2010; Weake and Workman, 2010). The main building block of chromatin is the nucleosome (Oudet et al., 1975), the core of which consists of 146-147 bp of DNA and a histone octamer of two each of the four core histones $\mathrm{H} 2 \mathrm{~A}, \mathrm{H} 2 \mathrm{~B}, \mathrm{H} 3$, and H4. The DNA is wrapped around the histones to form 1.65-1.67 turns of left-handed supercoils (Davey et al., 2002; Luger et al., 1997). The main body of chromatin fibers has nucleosome cores, core-connecting linker DNAs (Felsenfeld and Groudine, 2003; Simpson, 1978), and a specific complement of associated proteins such as the linker histone H1, localized at DNA entry and exit sites protruding from the nucleosome core, and architectural proteins (McBryant et al., 2006, 2010; Thoma et al., 1979). The simplest form of chromatin is the "beads-on-a-string" structures (10 $\mathrm{nm}$ fibers) formed by nucleosomes and 
linker DNAs (Thoma et al., 1979). The secondary packaging level of chromatin is thought to be $30 \mathrm{~nm}$ fibers, which have been observed in thin sections of intact cells and whole mount preparations (Igo-Kemenes et al., 1982; Kornberg, 1977; Richmond and Widom, 2000; and references therein). High ionic strength and the presence of linker histones can fold $10 \mathrm{~nm}$ fibers into $30 \mathrm{~nm}$ fibers in vitro (Huynh et al., 2005). However, the structures and higher order organization remain uncertain and various models have been proposed (Finch and Klug, 1976; Robinson et al., 2006; Schalch et al., 2005; Williams et al., 1986; Woodcock et al., 1984). Furthermore, recently a considerable controversy has arisen regarding the existence of $30 \mathrm{~nm}$ fibers in vivo (Eltsov, 2008; Fussner et al., 2011; Nishino et al., 2012).

\section{Nucleosomes can self-assemble}

Our recent study (Nishikawa and Ohyama, 2013) strongly suggested that nucleosomes have sequence-dependent self and non-self discrimination properties, and can self-assemble. We constructed oligonucleosomes using DNA octamers or tetramers and histone cores. Each of the original DNA fragments had 177 or 209 base pairs and contained a clone of Xenopus 5S rDNA nucleosome positioning sequence or those of artificial DNA sequences designated 601 and 603. The latter two clones were selected to contain strong nucleosome positioning sequences with high affinity for histone octamers (Lowary and Widom, 1998). We used various arrangements of these nucleosomes. The resulting oligonucleosomes were then subjected to AFM observations. Interestingly, we found that the nucleosomes with identical sequences tended to associate with each other in the oligonucleosomes. However, the movement of nucleosomes in a nucleosomal array was restricted in this system. Therefore, to determine whether the same kind of association occurs in a system free from such a structural restriction, we also examined nucleosome-nucleosome interactions using bead-fixed mononucleosomes and Alexa 555-labeled mononucleosomes. In this system, association was monitored as the increase of fluorescence intensity on the beads. This assay clearly showed that associations between the same nucleosome species were preferred over those between different species. Based on the topological aspects, the contact points in the association seem to reside on the core histone proteins (Fig. 2). It was speculated that the mechanisms underlying the selfassembly of homologous dsDNAs and those mediating the self-assembly of homologous nucleosomes may be different (Nishikawa and Ohyama, 2013).

\section{DNA self-assembly and nucleosome self-assembly may be key players in presynaptic alignment}

The pairing of homologous chromosomes begins during the late leptotene and early zygotene phases of meiosis. At first, homologous chromosomes are brought into close vicinity by some mechanism. In fission yeast, noncoding RNA seems to play this role. A recent study showed that even when homologous chromosomes are apart from each other, they can interact with assistance from noncoding RNA in fission yeast (Ding et al., 2012). Transcriptional machinery 


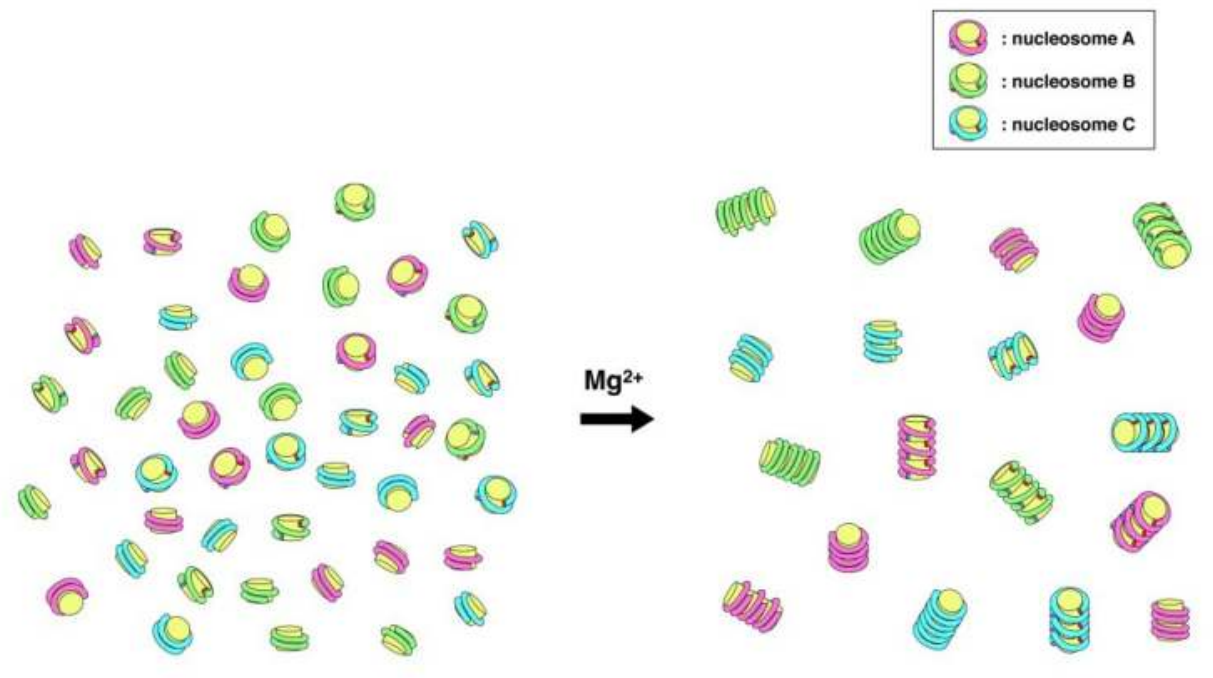

Figure 2. Nucleosomes have sequence-dependent self and non-self discrimination properties. Our study suggested that the contact points in the association of nucleosomes reside on the core histone proteins (Nishikawa and Ohyama, 2013).

proteins, cohesin, insulator or Polycomb proteins, may also play a similar role (Cook, 1997; McKee, 2004; Blumenstiel et al., 2008). Subsequently, homologous chromosomes recognize each other securely and align (pair), in preparation for synapsis. This stage is referred to as presynaptic alignment, and it may involve weak, homologous, paranemic DNA/DNA interactions (Stack and Anderson, 2001). The finding of the self-assembling property of DNA seems to substantiate this hypothesis. However, "paranemic DNA/DNA interactions" should be reconceptualized as "paranemic chromatin fiber/chromatin fiber interactions", because the DNA sequence-dependent self-assembling property of nucleosomes has also been proved to exist. In this discussion, a chromatin fiber is the $10 \mathrm{~nm}$ fiber, or the beads-on-a-string chromatin fiber.

In mammals, plants and fungi, the recombination pathway is often functionally interdigitated with presynaptic alignments and synapsis. Namely, in some organisms, meiotic doublestranded break (DSB) formation and recombination are required for these processes (Zickler, 2006; Zetka, 2009). On the other hand, in Drosophila and C. elegans, these processes proceed in the absence of DSBs and recombination (McKim et al., 1998; Dernburg et al. 1998; Zetka, 2009). Thus, DNA self-assembly and nucleosome self-assembly may be the essential mechanism of at least presynaptic alignment of the latter group (Fig. 3). Furthermore, they may also function in SC formation. 


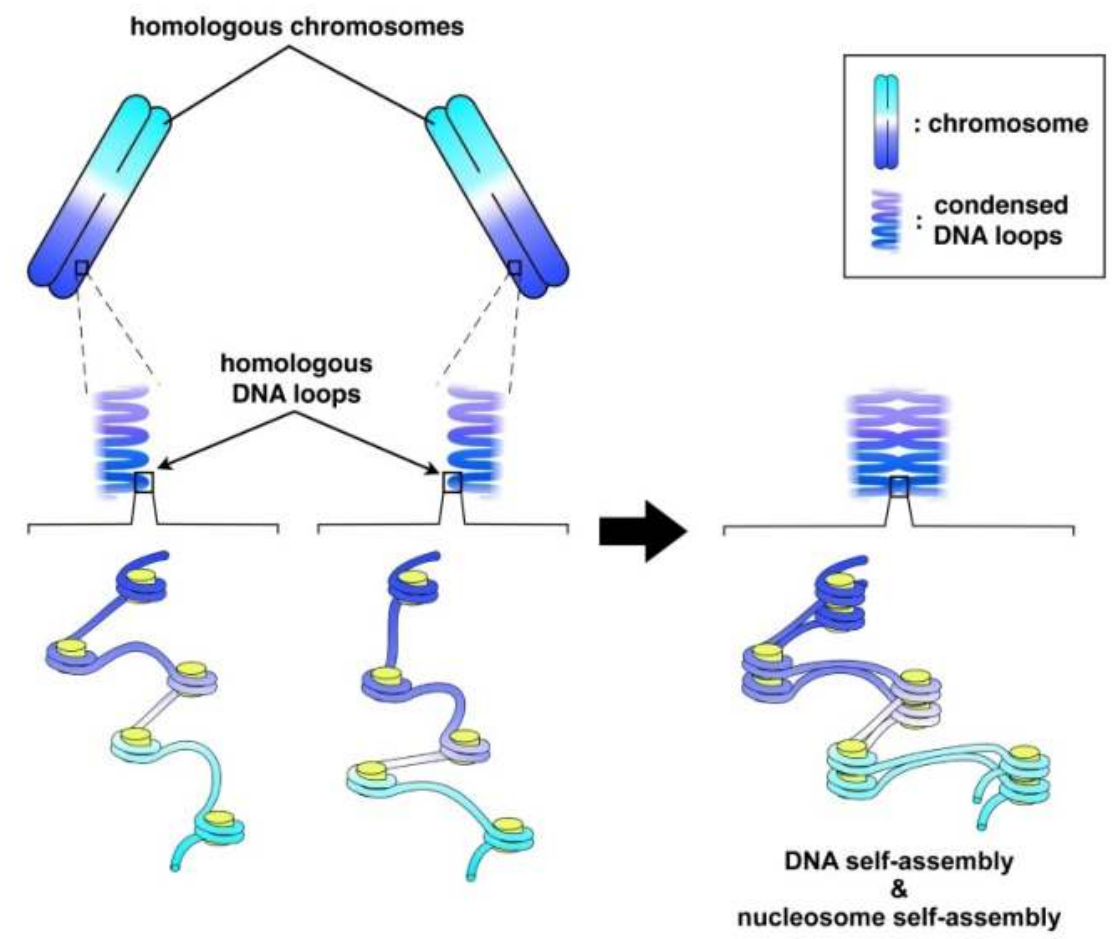

Figure 3. Schematic representation of DNA self-assembly and nucleosome self-assembly in paired homologous chromosomes.

\section{Conclusion}

In this chapter, we have described that both DNA and nucleosomes have sequence-dependent self and non-self discrimination properties, and can self-assemble. These properties of DNA and nucleosomes seem to be the key mediators of presynaptic alignment in some organisms. We hypothesize that the attractive force facilitating DNA self-assembly and nucleosome selfassembly is widely employed in many biological processes, including not only meiotic chromosome pairing but also somatic chromosome pairing, such as polytene chromosome formation in Diptera, transvection and other similar phenomena.

\section{Acknowledgements}

We acknowledge the support of the Ministry of Education, Culture, Sports, Science \& Technology - Japan (MEXT) to T.O. 


\section{Author details}

Jun-ichi Nishikawa ${ }^{1}$, Yasutoshi Shimooka² and Takashi Ohyama ${ }^{2 *}$

*Address all correspondence to: ohyama@waseda.jp

1 Department of Biology, Faculty of Education and Integrated Arts and Sciences, Waseda University, Shinjuku-ku, Tokyo, Japan

2 Integrative Bioscience and Biomedical Engineering, Graduate School of Science and Engineering, Waseda University, Shinjuku-ku, Tokyo, Japan

\section{References}

[1] Asakura, S. (1968). A kinetic study of in vitro polymerization of flagellin. J. Mol. Biol. , 35, 237-239.

[2] Baldwin, G. S, Brooks, N. J, Robson, R. E, Wynveen, A, Goldar, A, Leikin, S, Seddon, J. M, \& Kornyshev, A. A. (2008). DNA double helices recognize mutual sequence homology in a protein free environment. J. Phys. Chem. B , 112, 1060-1064.

[3] Blumenstiel, J. P, Fu, R, Theurkauf, W. E, \& Hawley, R. S. (2008). Components of the RNAi machinery that mediate long-distance chromosomal associations are dispensable for meiotic and early somatic homolog pairing in Drosophila melanogaster. Genetics , 180, 1355-1365.

[4] Cook, P. R. (1997). The transcriptional basis of chromosome pairing. J. Cell Sci. , 110, 1033-1040.

[5] Danilowicz, C, Lee, C. H, Kim, K, Hatch, K, Coljee, V. W, Kleckner, N, \& Prentiss, M. (2009). Single molecule detection of direct, homologous, DNA/DNA pairing. Proc. Natl. Acad. Sci. USA, 106, 19824-19829.

[6] Davey, C. A, Sargent, D. F, Luger, K, Maeder, A. W, \& Richmond, T. J. (2002). Solvent mediated interactions in the structure of the nucleosome core particle at $1.9 \AA$ resolution. J. Mol. Biol. , 319, 1097-1113.

[7] Dernburg, A. F, Mcdonald, K, Moulder, G, Barstead, R, Dresser, M, \& Villeneuve, A. M. (1998). Meiotic recombination in C. elegans initiates by a conserved mechanism and is dispensable for homologous chromosome synapsis. Cell , 94, 387-398.

[8] Ding, D. Q, Okamasa, K, Yamane, M, Tsutsumi, C, Haraguchi, T, Yamamoto, M, \& Hiraoka, Y. (2012). Meiosis-specific noncoding RNA mediates robust pairing of homologous chromosomes in meiosis. Science , 336, 732-736.

[9] Eltsov, M. MacLellan, K.M., Maeshima, K., Frangakis, A.S. and Dubochet, J. ((2008). Analysis of cryo-electron microscopy images does not support the existence of 30-nm 
chromatin fibers in mitotic chromosomes in situ. Proc. Natl. Acad. Sci. USA, 105, 19732-19737.

[10] Felsenfeld, G, \& Groudine, M. (2003). Controlling the double helix. Nature , 421, 448-453.

[11] Finch, J. T, \& Klug, A. (1976). Solenoidal model for superstructure in chromatin. Proc. Natl. Acad. Sci. USA , 73, 1897-1901.

[12] Fraenkel-conrat, H, \& Williams, R. C. (1955). Reconstitution of active tobacco mosaic virus from its inactive protein and nucleic acid components. Proc. Natl. Acad. Sci. USA, 41, 690-698.

[13] Fraser, R. D. MacRae, T.P. and Suzuki, E. ((1976). Structure of the alpha-keratin microfibril. J. Mol. Biol. , 108, 435-452.

[14] Fussner, E, Ching, R. W, \& Bazett-jones, D. P. (2011). Living without 30 nm chromatin fibers. Trends Biochem. Sci. , 36, 1-6.

[15] Horváth, I, Király, C, \& Szerb, J. (1949). Action of cardiac glycosides on the polymerization of actin. Nature 164, 792.

[16] Huynh, V. A, Robinson, P. J. J, \& Rhodes, D. (2005). A method for the in vitro reconstitution of a defined "30 nm" chromatin fibre containing stoichiometric amounts of the linker histone. J. Mol. Biol. , 345, 957-968.

[17] Igo-kemenes, T, Hörz, W, \& Zachau, H. G. (1982). Chromatin. Annu. Rev. Biochem. , $51,89-121$.

[18] Inoue, S, Sugiyama, S, Travers, A. A, \& Ohyama, T. (2007). Self-assembly of doublestranded DNA molecules at nanomolar concentrations. Biochemistry , 46, 164-171.

[19] Jehle, H. (1950). Specificity of Interaction Between Identical Molecules. Proc. Natl. Acad. Sci. USA , 36, 238-246.

[20] Jehle, H. (1963). Intermolecular forces and biological specificity. Proc. Natl. Acad. Sci. USA , 50, 516-524.

[21] Kornberg, R. D. (1977). Structure of chromatin. Annu. Rev. Biochem. , 46, 931-954.

[22] Kornyshev, A. A, \& Leikin, S. (2001). Sequence recognition in the pairing of DNA duplexes. Phys. Rev. Lett. , 86, 3666-3669.

[23] Kushner, D. J. (1969). Self-assembly of biological structures. Bacteriol. Rev. , 33, 302-345.

[24] Lowary, P. T, \& Widom, J. (1998). New DNA sequence rules for high affinity binding to histone octamer and sequence-directed nucleosome positioning. J. Mol. Biol. , 276, 19-42. 
[25] Luger, K, Mader, A. W, Richmond, R. K, Sargent, D. F, \& Richmond, T. J. (1997). Crystal structure of the nucleosome core particle at $2.8 \AA$ resolution. Nature , 389, 251-260.

[26] Mcbryant, S. J, Adams, V. H, \& Hansen, J. C. (2006). Chromatin architectural proteins. Chromosome Res. , 14, 39-51.

[27] Mcbryant, S. J, Lu, X, \& Hansen, J. C. (2010). Multifunctionality of the linker histones: an emerging role for protein-protein interactions. Cell Res. , 20, 519-528.

[28] Mcgavin, S. (1971). Models of specifically paired like (homologous) nucleic acid structures. J. Mol. Biol. , 55, 293-298.

[29] Mckee, B. D. (2004). Homologous pairing and chromosome dynamics in meiosis and mitosis. Biochim. Biophys. Acta , 1677, 165-180.

[30] Mckim, K. S, Green-marroquin, B. L, Sekelsky, J. J, Chin, G, Steinberg, C, Khodosh, R, \& Hawley, R. S. (1998). Meiotic synapsis in the absence of recombination. Science, $279,876-878$.

[31] Miki-noumura, T, \& Mori, H. (1972). Polymerization of tubulin: the linear polymer and its side-by-side aggregates. J. Mechanochem. Cell Motil. , 1, 175-188.

[32] Mueller, P, Rudin, D. O, Tien, H. T, \& Wescott, W. C. (1962). Reconstitution of cell membrane structure in vitro and its transformation into an excitable system. Nature, 194, 979-980.

[33] Nishikawa, J, \& Ohyama, T. (2013). Selective association between nucleosomes with identical DNA sequences. Nucleic Acids Res. , 41, 1544-1554.

[34] Nishino, Y, Eltsov, M, Joti, Y, Ito, K, Takata, H, Takahashi, Y, Hihara, S, Frangakis, A. S, Imamoto, N, Ishikawa, T, \& Maeshima, K. (2012). Human mitotic chromosomes consist predominantly of irregularly folded nucleosome fibres without a 30-nm chromatin structure. EMBO J. , 31, 1644-1653.

[35] Oosawa, F, \& Asakura, S. (1975). Thermodynamics of the Polymerization of Protein (London: Academic Press).

[36] Oudet, P, Gross-bellard, M, \& Chambon, P. (1975). Electron microscopic and biochemical evidence that chromatin structure is a repeating unit. Cell , 4, 281-300.

[37] Perham, R. N. (1975). Self-assembly of biological macromolecules. Philos. Trans. R. Soc. Lond. B , 272, 123-136.

[38] Ransom, M, Dennehey, B. K, \& Tyler, J. K. (2010). Chaperoning histones during DNA replication and repair. Cell , 140, 183-195.

[39] Richmond, T. J, \& Widom, J. (2000). Nucleosome and chromatin structure. In Chromatin Structure and Gene Expression, S.C.R. Elgin, and J.L. Workman, eds. (New York: Oxford University Press) , 1-23. 
[40] Robinson, P. J. J, Fairall, L, Huynh, V. A. T, \& Rhodes, D. (2006). EM measurements define the dimensions of the "30-nm" chromatin fiber: Evidence for a compact, interdigitated structure. Proc. Natl. Acad. Sci. USA , 103, 6506-6511.

[41] Schalch, T, Duda, S, Sargent, D. F, \& Richmond, T. J. (2005). X-ray structure of a tetranucleosome and its implications for the chromatin fibre. Nature , 436, 138-141.

[42] Simpson, R. T. (1978). Structure of the chromatosome, a chromatin particle containing 160 base pairs of DNA and all the histones. Biochemistry , 17, 5524-5531.

[43] Stack, S. M, \& Anderson, L. K. (2001). A model for chromosome structure during the mitotic and meiotic cell cycles. Chromosome Res. , 9, 175-198.

[44] Svejstrup, J. Q. (2010). The interface between transcription and mechanisms maintaining genome integrity. Trends Biochem. Sci. , 35, 333-338.

[45] Terasaki, M, \& Rubin, H. (1985). Evidence that intracellular magnesium is present in cells at a regulatory concentration for protein synthesis. Proc. Natl. Acad. Sci. USA, 82, 7324-7326.

[46] Thoma, F, Koller, T. H, \& Klug, A. (1979). Involvement of histone H1 in the organization of the nucleosome and of the salt-dependent superstructures of chromatin. J. Cell Biol. , 83, 403-427.

[47] Watson, J. D, \& Crick, F. H. C. (1953). Molecular structure of nucleic acids: a structure for deoxyribose nucleic acid. Nature , 171, 737-738.

[48] Weake, V. M, \& Workman, J. L. (2010). Inducible gene expression: diverse regulatory mechanisms. Nat. Rev. Genet. , 11, 426-437.

[49] Whitesides, G. M, \& Grzybowski, B. (2002). Self-assembly at all scales. Science , 295, 2418-2421.

[50] Williams, S. P, Athey, B. D, Muglia, L. J, Schappe, R. S, Gough, A. H, \& Langmore, J. P. (1986). Chromatin fibers are left-handed double helices with diameter and mass per unit length that depend on linker length. Biophys. J. , 49, 233-248.

[51] Woodcock, C. L. F, Frado, L. L. Y, \& Rattner, J. B. (1984). The higher-order structure of chromatin: evidence for a helical ribbon arrangement. J. Cell Biol. , 99, 42-52.

[52] Yos, J. M, Bade, W. L, \& Jehle, H. (1957). Specificity of the London-Eisenschitz Wang force. Proc. Natl. Acad. Sci. USA , 43, 341-346.

[53] Zetka, M. (2009). Homologue pairing, recombination and segregation in Caenorhabditis elegans. In Meiosis (Genome Dynamics R. Benavente, and J.-N. Volff, eds. (Basel: Karger) , 5, 43-55.

[54] Zickler, D. (2006). From early homologue recognition to synaptonemal complex formation. Chromosoma , 115, 158-174. 
